\title{
Q Iron Status and Metabolic Syndrome in Patients with Non-Alcoholic Fatty Liver Disease
}

\author{
Mohammad Ebrahim Ghamarchehreh ${ }^{1}$, Nematollah Jonaidi-Jafari², Mohammad Bigdeli ${ }^{1}$, \\ Hossein Khedmat ${ }^{1 *}$, Amin Saburi ${ }^{3}$
}

1. Baqiyatallah Research Center for Gastroenterology and Liver Diseases, Baqiyatallah University of Medical Sciences, Tehran, Iran

2. Health Research Center, Baqiyatallah University of Medical Sciences, Tehran, Iran

3. Birjand Atherosclerosis and Coronary Artery Research Center, Birjand University of Medical Sciences, Birjand, Iran \& Chemical Injuries Research Center, Baqiyatallah University of Medical Sciences, Tehran, Iran

\footnotetext{
* Corresponding Author: Hossein Khedmat, MD Baqiyatalah Research Center for Gastroenterology and Liver Disease, Baqiyatallah University of Medical Sciences, Tehran, Iran

Telefax: + 982188600067

Email: md.researcher@yahoo.com

Received: 19 Apr. 2015

Accepted: 10 Sep. 2015
}

\section{ABSTRACT}

\section{BACKGROUND}

A hypothesis has been presented about the role of serum iron, ferritin and transferrin saturation among patients with non-alcoholic fatty liver disease (NAFLD) and resistance to insulin (metabolic syndrome [MetS]), but there is much controversy. This study aimed at investigating the level of serum iron and demographic characteristics in patients with NAFLD with or without MetS.

\section{METHODS}

A case-control study was conducted on patients with elevated liver enzymes referring to Baqiyatallah clinic, Tehran, Iran during 2010-2011. After ruling out other causes of increased aminotransferases and approving the diagnosis of NAFLD, the patients were divided into two groups of with or without MetS. Then, the individuals' demographic, sonographic, and laboratory characteristics were recorded.

\section{RESULTS}

This research included 299 patients suffering from NAFLD who were divided into MetS $(n=143 ; 47.8 \%)$ and non-MetS $(n=156 ; 52.2 \%)$ groups. The age, systolic and diastolic blood pressure, body mass index, waist/hip ratio, glucose tolerance test, serum insulin, C. peptide, triglyceride, and HB A1c were different between MetS and non-MetS groups $(p<0.05)$. There was no significant difference in serum iron and ferritin levels between the two groups, however, a significant correlation was found between serum ferritin and alanine transaminase $(p=0.005)$ and also aspartate aminotransferase $(p=0.032)$.

\section{CONCLUSION}

Our findings did not show a significant relationship between iron, in free or storage form, and the presence of MetS among patients with NAFLD, but serum ferritin can correlate with hepatocytes injuries indicated by raised aminotransferases. Nevertheless, to clarify this relationship further molecular, genomic, and histopathological studies are required.

\section{KEYWORDS}

Non-alcoholic fatty liver disease; Metabolic syndrome; Iron; Ferritin

Please cite this paper as:

Ghamar-Chehreh ME, Jonaidi-Jafari N, Bigdeli M, Khedmat H, Saburi A. Iron Status and Metabolic Syndrome in Patients with Non-Alcoholic Fatty Liver Disease Middle East J Dig Dis 2015;8:31-8. DOI :10.15171/mejdd.2016.04

\section{INTRODUCTION}

Metabolic Syndrome (MetS) or syndrome of resistance to insulin involves metabolic abnormalities indicated by central obesity, hypertriglyceridemia, decreased high density lipoprotein (HDL), hypergly- 
cemia, obstructive sleep apnea, and hypertension. ${ }^{1-3}$ According to theAdult Treatment Panel III (ATP III) report, the prevalence of MetS is about $22 \%$, which is increased by age. ${ }^{4}$ Many metabolic abnormalities such as non-alcoholic fatty liver disease (NAFLD) and steatohepatitis are observed along with MetS. 5,6 "NAFLD is a hepatic manifestation of metabolic syndrome; it is closely related to other clinical features of metabolic syndrome". ${ }^{7}$ NAFLD is an inflammatory liver disease in which fat accumulates in hepatocytes and results in increased number of inflammatory cells in liver tissue. ${ }^{8}$ NAFLD is the most common liver disease in developed countries with the incidence rate of $20-40 \%$, however, its prevalence is rising in developing countries such as Asian nations. ${ }^{9}$ Most frequently, NAFLD is asymptomatic and is detected when a physician performs precise laboratory or ultrasound studies for checkup or increase in aminotransferases occurs. ${ }^{7,10}$

This disease was first observed among middle aged obese men with diabetes mellitus (DM) but recently it is believed that non-obese and non-diabetic men with iron overload could be predisposed to this disease. ${ }^{11}$ Therefore, currently the role of serum iron, ferritin, and transferrin in this disease is more studied. ${ }^{12,13}$ Also, a newly diagnosed condition of liver called "insulin resistance-hepatic iron overload IR-HIO" is observed in patients with hyper ferritinemia and normal transferrin without mutation in gene of hemochromatosis. Although patients with IR-HIO suffer from a wide verity of metabolic disorders, the relationship between IR$\mathrm{HIO}$ and NAFLD is not yet definite. ${ }^{14}$

On the other hand, there are several hypotheses about the pathophysiology of the relationship between NAFLD and MetS. One of the most acceptable hypotheses is insulin resistance indicated by postprandial ineffective hyperinsulinemia and subsequently postprandial hyperglycemia and increase in free fatty acid. ${ }^{15}$ Furthermore, oxidant and antioxidant imbalance is another trigger suggested for justifying this relationship whereas ferritin is an acute phase reactant. ${ }^{16}$ Both mechanisms could support the hypothesis on the role of iron or iron carriers in this correlation between MetS and NAFLD.
In this study we assessed clinical and para-clinical evidence for this relationship.

\section{MATERIALS AND METHODS}

A case-control study was conducted between 2010 and 2011 among patients with elevated aminotransferases referred to Baqiyatallah clinic, Tehran, Iran. The diagnosis of NAFLD was confirmed after ruling out other causes of elevated aminotransferases such as viral hepatitis (by testing HBS Ag, HBS $\mathrm{Ab}, \mathrm{HBC} \mathrm{Ab}$, and HCV Ab), alcohol consumption, infectious diseases, and other medical conditions, which could affect liver function test. Based on the following criteria (table 1), the patients were divided into two groups with MetS (MetS group) or without MetS (non-MetS group). Guideline of ATP version 3 was used for diagnosis of MetS. ${ }^{17}$ Clinical and paraclinical assessments were done. The clinical assessment, included demographic and anthropometric characteristics, and physical examination were done by a clinical practitioner.

Paraclinical studies were also performed consisting of ultrasonography and lab tests aspartate aminotransferase(AST), Alanine transaminase(ALT),Low-density lipoprotein (LDL), High-density lipoprotein (HDL), triglyceride (TG), cholesterol, fasting blood sugar (FBS), ferritin, and serum iron), which were done in the laboratory of Baqiyatallah Hospital after 12 hours fasting. Diagnosis and grading of NAFLD (Diffuse hepatic steatosis) based on ultrasonography was determined by a radiologist according to the following guideline;

1 - increased echogenicity and beam attenuation, which is known as a diffuse hyperechoic texture (bright liver)

2- Increased echo texture compared with kidney (liver and renal cortex have normally a similar echogenicity)

3- vascular blurring (absence of the normal echogenic walls of the portal veins and hepatic veins).

Using the sample size formula shown below, the sample size was calculated as 280 cases, while $\mathrm{d}=0.05, p=0.68$, and $\alpha=0.05$.

for aminotransferases was $40 \mathrm{mg} / \mathrm{dL}$. For serum 
iron it was $190 \mu \mathrm{g} / \mathrm{dL}$ for men and $175 \mu \mathrm{g} / \mathrm{dL}$ for women. Cut point for serum ferritin was $300 \mu \mathrm{g} / \mathrm{dL}$ for men and $200 \mu \mathrm{g} / \mathrm{dL}$ for women. Chi2 (or Fisher

$n=\frac{\left(Z_{1-x / 2}\right)^{2} \times P(1-P)}{d^{2}}$

exact test), independent $t$ test, one way ANOVA, and Pearson (or spearman) tests were used for univariate analysis in using SPSS software version 16 . P value less than 0.05 were considered as statistically significant. Finally, variables with $\mathrm{P}$ value less than 0.2 was entered in a model of logistic regression.

\section{RESULTS}

A total of 299 patients (198 men and 101 women) with NAFLD were included and were divided intoMetS $(n=143 ; 47.8 \%)$ and Non-MetS $(n=156$; $52.2 \%)$ groups. The mean age of the patients was $44.99 \pm 12.77$ years. The baseline characteristics of each group are illustrated in table 2 and 3 . There is a significant difference between mean age and sex ratio in the two groups $(p=0.001)$.

As expected, the age, systolic and diastolic blood pressure, body mass index (BMI), waist/hip ratio, glucose tolerance test (GTT), serum insulin, C. peptide, TG, and hemoglobin A1c (HbAlc) were different between MetS and non-MetS groups $(p<0.05)$. Moreover, the history of diabetes mellitus, hypertension, and coronary artery disease was significantly different between the groups $(p<0.05)$. There was no significant difference in grade of disease according to the ultrasound study, aminotransferases and bilirubin levels between MetS and non-MetS groups. After adjusting for confounder variables such as age, we found no differences in ALT $(p=0.557)$ and AST level $(p=0.205)$ among the two groups. However, a significant correlation was found between serum ferritin and ALT $(p=0.005)$ and also $\operatorname{AST}(p=0.032)$.

Despite adjusting the confounding role of sex on serum ferritin and iron, we could not find any significant difference in serum iron and ferritin level between the two groups (table 4 ). In a regression model, only sex $(p<0.001$, Exp B: 3.912 , CI 95\%: $1.892-8.088)$, systolic blood pressure $(p=0.013$, Exp
Table1: Guideline of ATPIII 2005 (National Cholesterol Education Program; NCEP)

\section{NCEP' ${ }^{1}$ ATP3}

3 items and more;

abdominal circumference $\geq 102 \mathrm{~cm}$ for men and $\geq 88 \mathrm{~cm}$ for women

Triglyceride $\geq 150 \mathrm{mg} / \mathrm{dl}$ or under treatment with lipid lowering agents

Blood pressure $\geq 130$ systolic or $\geq 85$ diastolic or under treatment for hypertension

FBS $\geq 100 \mathrm{mg} / \mathrm{dl}$ or under treatment for diabetes mellitus

$\mathrm{HDL} \geq 40 \mathrm{mg} / \mathrm{dl}$ in men or $\geq 50 \mathrm{mg} / \mathrm{dl}$ in women or under treatment for decreased HDL

B: [1.040] CI 95\%: 1.008-1.072), BMI ( $p=0.001$, Exp B: 1.158, CI 95\%: 1.061-1.264), DM ( $p=0.007$, Exp B: 4.305, CI 95\%: 1.481-12.509), total iron binding capacity (TIBC) ( $p=0.017$, EXP B: 1.007 , CI 95\%: 1.001-1.017), hemoglobin $(p=0.017, \mathrm{EXP}$ B: 1.007, CI 95\%: 1.001-1.017), TG ( $p=0.017, \mathrm{EXP}$ B: 1.007, CI 95\%: 1.001-1.017), HDL ( $p=0.017$, EXP B: 1.007, CI 95\%: 1.001-1.017), alkaline phosphatase (ALP) $(p=0.017$, EXP B: 1.007, CI 95\%: $1.001-1.017)$, AST $(p=0.017$, EXP B: 1.007 , CI 95\%: 1.001-1.017), ALT ( $p=0.017$, EXP B: 1.007 , CI 95\%: 1.001-1.017), and serum insulin ( $p=0.017$, EXP B: 1.007, CI 95\%: 1.001-1.017) could predict MetS in patients with NAFLD.

\section{DISCUSSION}

Given the results of this study, no significant relationship was observed between serum iron and presence of MetS in patients with NAFLD. Also, the same results were obtained for serum ferritin. In other words, there is no prominent difference in the role of iron (both serum iron and ferritin as a form of iron for storage) in presence of NAFLD in both groups.

A hypothesis has been presented on the role of iron especially iron deposit in liver in patients with NAFLD on resistance to insulin, however, there is much controversy in determination of definite mechanism. ${ }^{18}$

In late 1990s, two studies posed the hypothesis about irrespective resistance to insulin among patients with hepatic iron overload, which was indicated by hyperferritinemia and normal transferrin. ${ }^{19}$ Mendler and colleagues demonstrated that 
Table 2: baseline characteristics (quantitative variable) in terms of groups

\begin{tabular}{|c|c|c|c|}
\hline Variables & NON-METS & METS & $p$-value (r) \\
\hline Age & $42.65 \pm 13.08$ & $47.45 \pm 11.87$ & $0.001(-3.245)$ \\
\hline SBP & $119.01 \pm 11.77$ & $125.09 \pm 17.10$ & $0.001(-3.553)$ \\
\hline DBP & $79.01 \pm 6.19$ & $82.05 \pm 6.47$ & $0.001(-3.504)$ \\
\hline Weight & $83.92 \pm 16.64$ & $85.37 \pm 14.77$ & 0.776 \\
\hline Height & $168.95 \pm 13.49$ & $166.12 \pm 10.39$ & 0.556 \\
\hline Abdominal Girth & $101.47 \pm 9.98$ & $107.42 \pm 11.34$ & 0.374 \\
\hline Pelvic Girth & $102.344 \pm 9.69$ & $105.83 \pm 1043$ & 0.955 \\
\hline BMI & $28.56 \pm 3.46$ & $30.95 \pm 4.69$ & $0.000(-4.66)$ \\
\hline Waist/hip ratio & $1.25 \pm 0.48$ & $1.49 \pm 0.60$ & $0.001(-3.514)$ \\
\hline Pack/year & $3.20 \pm 6.69(15)$ & $8.71 \pm 18.23(7)$ & 0.464 \\
\hline GTT & $116.24 \pm 49.06$ & $145.52 \pm 66.52$ & $0.000(-3.95)$ \\
\hline Serum Insulin & $10.88 \pm 5.69$ & $14.54 \pm 7.46$ & $0.000(-0.450)$ \\
\hline C. peptide & $2.49 \pm 1.07$ & $3.02 \pm 0.99$ & $0.000(-3.93)$ \\
\hline HBA1C & $5.76 \pm 0.91$ & $7.00 \pm 6.40$ & $0.033(0.215)$ \\
\hline Cholesterol & $199.49 \pm 45.54$ & $196.86 \pm 43.62$ & 0.905 \\
\hline TB & $175.78 \pm 114.05$ & $240.37 \pm 124.54$ & $0.000(-4.63)$ \\
\hline HDL & $117.49 \pm 36.33$ & $43.23 \pm 9.88$ & $0.000(4.61)$ \\
\hline LDL & $117.49 \pm 38.33$ & $110.91 \pm 34.13$ & 0.124 \\
\hline Total Bilirubin & $1.08 \pm 0.615$ & $1.04 \pm 0.54$ & 0.644 \\
\hline Direct Bilirubin & $5.95 \pm 1.33$ & $6.10 \pm 168$ & 0.455 \\
\hline ALP & $188.32 \pm 57.38$ & $197.90 \pm 67.75$ & 0.205 \\
\hline $\mathrm{AST}$ & $30.94 \pm 15.78$ & $32.87 \pm 22.67$ & 0.404 \\
\hline ALT & $48.03 \pm 32.70$ & $46.31 \pm 38.23$ & 0.678 \\
\hline ALB & $4.69 \pm 0.85$ & $4.56 \pm 0.43$ & 0.169 \\
\hline PT & $12.54 \pm 0.90$ & $12.53 \pm 1.49$ & 0.964 \\
\hline $\mathrm{TSH}$ & $2.31 \pm 2.01$ & $2.33 \pm 3.11$ & 0.946 \\
\hline T3 & $1.97 \pm 6.71$ & $1.29 \pm 0.37$ & 0.266 \\
\hline $\mathrm{T} 4$ & $92.42 \pm 21.79$ & $89.61 \pm 18.76$ & 0.272 \\
\hline Serum Feritine & $163.78 \pm 246.33$ & $151.54 \pm 205.82$ & 0.682 \\
\hline Serum Iron & $108.92 \pm 39.37$ & $151.86 \pm 490.62$ & 0.305 \\
\hline TIBC & $317.84 \pm 59.20$ & $334.45 \pm 59.99$ & $0.032(-2.15)$ \\
\hline Ceruloplomin & $33.24 \pm 51.82$ & $24.32 \pm 10.68$ & 0.501 \\
\hline $\mathrm{HB}$ & $15.27 \pm 1.47$ & $14.52 \pm 1.44$ & $0.0001(4.28)$ \\
\hline $\mathrm{MCV}$ & $85.03 \pm 7.70$ & $86.06 \pm 5.32$ & 0.199 \\
\hline PIT & $238.67 \pm 56.790$ & $276.33 \pm 30.391$ & 0.160 \\
\hline
\end{tabular}

"patients with unexplained hepatic iron overload are characterized by a mild to moderate iron burden and the nearly constant association of an insulin resistance syndrome irrespective of liver damage." 20 Moirand and co-workers also presented this condition as a new non-HLA-linked iron overload syndrome, which suggests a relationship between iron excess and an abnormal metabolic condition in- cluding obesity, hyperlipidemia, abnormal glucose metabolism, or hypertension known as MetS. ${ }^{21}$ An epidemiological finding that "the dysmetabolic iron overload syndrome is detected in about one third of patients with NAFLD and the MetS" and also a therapeutic-based approach that showed decreased metabolic alterations and liver enzymes in NAFLD patients undergone iron depletion by phlebotomy 
Table3: baseline characteristics (qualitative variable) in terms of groups

\begin{tabular}{|c|c|c|c|c|c|}
\hline Item & Sub group & NON-METS & METS & $p$-value & OR (CI) \\
\hline \multirow{2}{*}{ Gender } & M & $76.6 \%$ & $47.5 \%$ & \multirow{2}{*}{$<0.001$} & \multirow{2}{*}{$\begin{array}{c}1.614 \\
(1.32-1.96)\end{array}$} \\
\hline & $\mathrm{F}$ & $23.4 \%$ & $52.5 \%$ & & \\
\hline \multirow{4}{*}{ Education } & Illiterate & $0 \%$ & $0.7 \%$ & \multirow{4}{*}{$<0.001$} & \multirow{4}{*}{ - } \\
\hline & Under D & $20.3 \%$ & $45.3 \%$ & & \\
\hline & Diploma & $73.9 \%$ & $50.4 \%$ & & \\
\hline & Scholar & $5.9 \%$ & $3.6 \%$ & & \\
\hline \multirow{2}{*}{ Symptoms } & + & $24 \%$ & $39.6 \%$ & \multirow{2}{*}{0.004} & \multirow{2}{*}{$\begin{array}{c}0.607 \\
(0.429-0.860)\end{array}$} \\
\hline & - & $76 \%$ & $60.4 \%$ & & \\
\hline \multirow{4}{*}{ Sonography } & NL & $2.6 \%$ & $0.7 \%$ & \multirow{4}{*}{0.119} & \multirow{4}{*}{-} \\
\hline & Grade 1 & $51.3 \%$ & $40.3 \%$ & & \\
\hline & Grade 2 & $32.2 \%$ & $42.4 \%$ & & \\
\hline & Grade 3 & $13.8 \%$ & $16.5 \%$ & & \\
\hline \multirow{2}{*}{ DM } & + & $5.2 \%$ & $18 \%$ & \multirow{2}{*}{0.001} & \multirow{2}{*}{$\begin{array}{c}0.289 \\
(0.135-0.619)\end{array}$} \\
\hline & - & $49.8 \%$ & $82 \%$ & & \\
\hline \multirow{2}{*}{ CAD } & + & $96.8 \%$ & $3.2 \%$ & \multirow{2}{*}{0.030} & \multirow{2}{*}{$\begin{array}{c}0.347 \\
(0.127-0.947)\end{array}$} \\
\hline & - & $90.6 \%$ & $9.4 \%$ & & \\
\hline \multirow{2}{*}{ HTN } & + & $7.8 \%$ & $30.4 \%$ & \multirow{2}{*}{$<0.001$} & \multirow{2}{*}{$\begin{array}{c}0.256 \\
(0.141-0.466)\end{array}$} \\
\hline & - & $92.2 \%$ & $69.6 \%$ & & \\
\hline \multirow{2}{*}{ Hypothyroid } & + & $5.2 \%$ & $7.9 \%$ & \multirow{2}{*}{0.345} & \multirow{2}{*}{-} \\
\hline & - & $94.8 \%$ & $92.1 \%$ & & \\
\hline \multirow{2}{*}{ Smoking } & + & $9.7 \%$ & $5.8 \%$ & \multirow{2}{*}{0.205} & \multirow{2}{*}{ - } \\
\hline & - & $90.3 \%$ & $94.2 \%$ & & \\
\hline \multirow{2}{*}{ FX of DM } & + & $36.4 \%$ & $44.2 \%$ & \multirow{2}{*}{0.148} & \multirow{2}{*}{ - } \\
\hline & - & $63.6 \%$ & $55.8 \%$ & & \\
\hline \multirow{2}{*}{ INR } & $>2.5$ & $45 \%$ & $81.3 \%$ & \multirow{2}{*}{$<0.001$} & \multirow{2}{*}{$\begin{array}{c}0.554 \\
(0.452-0.677)\end{array}$} \\
\hline & $<2.5$ & $18.7 \%$ & $55 \%$ & & \\
\hline
\end{tabular}

Table 4: Report of serum iron and ferritin in term of gender

\begin{tabular}{lccccc}
\hline Item & Gender & MetS & Mean & SD & p-value \\
\hline \multirow{3}{*}{ Serum iron } & \multirow{2}{*}{ female } & yes & 101.29 & 44.45 & \\
\cline { 2 - 5 } & & no & 102.65 & 28.88 & \\
\cline { 2 - 5 } & \multirow{2}{*}{ male } & yes & 109.58 & 70.09 & \multirow{2}{*}{0.639} \\
\cline { 3 - 5 } & \multirow{2}{*}{ female } & no & 111.77 & 43.17 & \\
\cline { 3 - 5 } Serum ferritin & & yes & 106.52 & 44.46 & \multirow{2}{*}{0.004} \\
\cline { 2 - 4 } & \multirow{2}{*}{ male } & yes & 132.21 & 90.04 & \\
\cline { 3 - 5 } & & no & 139.41 & 95.78 & \multirow{2}{*}{0.952} \\
\hline
\end{tabular}

uphold this hypothesis..$^{22,23}$

Mechanisms of the relationship between iron accretion and insulin resistance, as the main pathogenesis of MetS, in patients with NAFLD have been poorly studied. Genetic factors, oxidative stress, cell toxicity, and genotoxicity can predis- pose iron deposits due to circulatory iron excess. These factors can induce iron storage in hepatocytes and Kupffer/sinusoidal cells. ${ }^{19,24}$ In molecular and histopathological point of view, Kupffer cells can accumulate iron via phagocytosis of necrotic hepatocytes, or probably erythrophagocytosis. ${ }^{24}$ 
Conversely in this study, a significant correlation was obtained between serum ferritin and ALT and also AST indicating for hepatocytes injuries by depositing fat. Also, we found higher levels of hemoglobin and TIBC in MetS group compared with non-MetS group. This finding is in accordance with the mentioned hypothesis. Regularly, these indexes correlate with red blood cell (RBC) count and also erythrophagocytosis. There are some pivotal factors that induce hepatic iron uptake such as deficiency of micronutrients, inflammation, dysregulation of iron trafficking molecules, and hyperinsulinemia. "Insulin stimulates cellular iron uptake through increased externalization of the transferring, receptor in adipocytes". ${ }^{19,25}$

On the other hand, in the adipocyte, the stored iron can affect insulin sensitivity of the cell. Both aforementioned mechanisms can cause unsuppressed lipolysis, and therefore liver is predisposed to fat storage. ${ }^{26}$ Stimulation of intestinal iron absorption, by hyperinsulinism and insulin resistance, is the other mechanism for the increase of body iron stores while in the present study we did not find any difference between the amount of storage form of iron, ferritin, between the two groups, ${ }^{14,27,28}$ although there was a difference in serum insulin levels between the two groups.

Given the findings of the present study in keeping with previous findings, TIBC (indicator for the transferrin activity) is the sole iron trafficking index that was different between the two groups. ${ }^{29}$ As expected, age, blood pressure, BMI, hip/waist ratio, prevalence of DM and coronary artery diseases (CAD), serum TG, and HDL were higher in MetS group compared with non-MetS group. It was previously clarified that patients with MetS with or without other medical conditions, such as NAFLD, are more frequently involved in DM, CAD, hypertension, obesity, and hyperlipidemia which is in line with our findings. Abdominal obesity indicated by high waist/hip ratio albeit is an important risk factor for metabolic disturbances, including fatty liver. ${ }^{30}$ After adjusting the confounders, we could not find a significant relationship between abdomi- nal fat levels and presence of MetS.

But international normalized ratio (INR) as a coagulation status index was different between the two groups. Usually coagulation defect occur when NAFLD leads to cirrhosis while none of the patients suffered from cirrhosis. The sonographic grade of NAFLD was not significantly different between MetS and non-MetS group. The role of iron in coagulation was previously declared ${ }^{31}$ however, we could not precisely associate these two with the difference in INR and iron level in the two groups.

One of the limitations of this study was the lack of pathological and genomic studies to compare MetS and non-MetS groups. To verify this comparison, liver biopsies would be helpful but this is ethically unfeasible since it is unnecessary in patients with uncomplicated NAFLD. The other limitation was lack of further immunological assessments such as inflammatory markers. Ferrannini demonstrated an association between hyperinsulinemia and the excess hepatic iron storage in inflammatory conditions. ${ }^{32}$ Over transcriptions of ferritin mRNA in macrophages due to inflammatory cytokine can be responsible for ferritin transfer to hepatocytes.

Consistent with our findings, previous studies that showed a prominent relationship between the presence of Mets and serum iron or ferritin in patients with NAFLD recommended searching for hepatic iron accumulation in biopsy samples of patients with NAFLD, even in cases with normal serum iron, ferritin, and transferrin. ${ }^{33}$ Conversely, Choi and colleagues, evaluated serum ferritin and aminotransferases among 994 post menaupose women. They found that women with MetS had a higher level of serum ferritin compared with women without MetS. ${ }^{34}$ Many other studies indicated a probable relationship between NAFLD as one of the manifestations of MetS and serum level of ferritin. ${ }^{35-38}$ But, consistent with our findings, Freixenet and co-workers also demonstrated that iron level had no correlation with existing DM and MetS. ${ }^{39}$ Moreover, this difference between the findings of various reports can be due to sociodemographic varieties. Therefore, if an adapted cut point is deter- 
mined for every population, a significant relationship may be obtained. Although we could not find a similar relationship, but we found that serum level of ferritin in patients with NAFLD and MetS was above 125-130.

Our findings did not show a significant relationship between iron, in free or storage form, and the presence of MetS among patients with NAFLD, but serum ferritin can correlate with hepatocytes injuries indicated by raised aminotransferases. Nevertheless, to clarify this relationship further molecular, genomic, and histopathological studies are required.

\section{ACKNOWLEDGEMENT}

The authors are grateful to all patients for their collaboration in this study. Also, the authors thank Baqiyatallah Research Center of Gastroenterology and Liver Diseases for supporting this survey financially.

\section{CONFLICT OF INTEREST}

The authors declare no conflict of interest related to this work.

\section{REFERENCES}

1. Cho LW. Metabolic syndrome. Singapore Med J 2011;52:779-85.

2. .El-Koofy NM, Anwar GM, El-Raziky MS, El-Hennawy AM, El-Mougy FM, El-Karaksy HM, et al. The association of metabolic syndrome, insulin resistance and nonalcoholic fatty liver diseasein overweight/obese children. Saudi J Gastroenterol 2012;18:44-9. DOI:10.4103/13193767.91738

3. Lewis JR, Mohanty SR. Nonalcoholic fatty liver disease: a review and update. Dig Dis Sci 2010;55:560-78. DOI:10.1007/s10620-009-1081-0.

4. Ford ES, Giles WH, Dietz WH. Prevalence of the metabolic syndrome among US adults: findings from the third National Health and Nutrition Examination Survey. JAMA 2002;287:356-9. DOI:10.1001/jama.287.3.356.

5. Marceau P, Biron S, Hould FS, Marceau S, Simard S, Thung $\mathrm{SN}$, et al. Liver pathology and the metabolic syndrome $\mathrm{X}$ in severe obesity. J Clin Endocrinol Metab 1999;84:1513-7.

6. Choi HK, Ford ES. Prevalence of the metabolic syndrome in individuals with hyperuricemia. Am J Med 2007;120:4427. DOI:http://dx.doi.org/10.1016/j.amjmed.2006.06.040.

7. Milic S, Stimac D. Nonalcoholic fatty liver disease/ steatohepatitis: epidemiology, pathogenesis, clinical presentation and treatment. Dig Dis 2012;30:158-62. DOI:10.1159/000336669.

8. Khedmat H, Taheri S. Non-alcoholic steatohepatitis: An update in pathophysiology, diagnosis and therapy. Hepat Mon 2011;11:74-85.

9. Amarapurkar DN, Hashimoto E, Lesmana LA, Sollano JD, Chen PJ, Goh KL. How common is non-alcoholic fatty liver disease in the Asia-Pacific region and are there 10cal differences? J Gastroenterol Hepatol 2007;22:788-93. DOI:10.1111/j.1440-1746.2007.05042.x.

10. Hajiaghamohammadi A, Ziaee A, Oveisi S, Masroor H. Effects of Metformin, Pioglitazone, and Silymarin Treatment on Non- Alcoholic Fatty Liver Disease: A Randomized Controlled Pilot Study. Hepat Mon 2012;12:1-6. DOI:10.5812/hepatmon.6099.

11. Dinneen SF, Silverberg JD, Batts KP, O’Brien PC, Ballard DJ, Rizza RA. Liver iron stores in patients with non-insulindependent diabetes mellitus. Mayo Clin Proc 1994;69:135. DOI:http://dx.doi.org/10.1016/S0025-6196(12)61605-x.

12. Machado M, Cortez-Pinto H. Nash, insulin resistance and iron. Liver Int 2006;26:1159-62. DOI:10.1111/j.14783231.2006.01394.x.

13. Sumida Y, Yoshikawa T, Okanoue T. Role of hepatic iron in non-alcoholic steatohepatitis. Hepatol Res 2009;39:21322. DOI:10.1111/j.1872-034X.2008.00442.x.

14. Le Guenno G, Chanseaume E, Ruivard M, Morio B, Mazur A. Study of iron metabolism disturbances in an animal model of insulin resistance. Diabetes Res Clin Pract 2007;77:363-70. DOI:http://dx.doi.org/10.1016/j.diabres.2007.02.004

15. Bush NC, Basu R, Rizza RA, Nair KS, Khosla S, Jensen MD. Insulin-Mediated FFA Suppression Is Associated with Triglyceridemia and Insulin Sensitivity Independent of Adiposity. J Clin Endocrinol Metab 2012;97:4130-8. DOI:10.1210/jc.2012-2285.

16. Morita M, Ishida N, Uchiyama K, Yamaguchi K, Itoh Y, Shichiri M, et al. Fatty liver induced by free radicals and lipid peroxidation. Free Radic Res 2012;46:758-65. DOI:1 0.3109/10715762.2012.677840.

17. McKenney JM. Update on the National Cholesterol Education Program Adult Treatment Panel III guidelines: getting to goal. Pharmacotherapy 2003;23:26S-33S. DOI: $10.1592 /$ phco.23.11.26S.32710.

18. O'Brien J, Powell LW. Non-alcoholic fatty liver disease: is iron relevant? Hepatol Int 2012;6:332-41. DOI:10.1007/ s12072-011-9304-9.

19. Manco M, Alisi A, Real JM, Equitani F, Devito R, Valenti $\mathrm{L}$, et al. Early interplay of intra-hepatic iron and insulin resistance in children with non-alcoholic fatty liver disease. $J$ Hepatol 2011;55:647-53. DOI:10.1016/j.jhep.2010.12.007.

20. Mendler MH, Turlin B, Moirand R, Jouanolle AM, Sapey $\mathrm{T}$, Guyader D, et al. Insulin resistance-associated hepatic iron overload. Gastroenterology 1999;117:1155-63. 
DOI:http://dx.doi.org/10.1016/S0016-5085(99)70401-4.

21. Moirand R, Mortaji AM, Loreal O, Paillard F, Brissot P, Deugnier Y. A new syndrome of liver iron overload with normal transferrin saturation. Lancet 1997;349:95-7. DOI:http://dx.doi.org/10.1016/S0140-6736(96)06034-5.

22. Dongiovanni P, Fracanzani AL, Fargion S, Valenti L. Iron in fatty liver and in the metabolic syndrome: a promising therapeutictarget. J Hepatol 2011;55:920-32. DOI:10.1016/j. jhep.2011.05.008.

23. Valenti L, Fracanzani AL, Dongiovanni P, Bugianesi E, Marchesini G, Manzini P, et al. Iron depletion by phlebotomy improves insulin resistance in patients with nonalcoholic fatty liver disease and hyperferritinemia: evidence from a case-control study. Am J Gastroenterol 2007;102:12518. DOI:10.1111/j.1572-0241.2007.01192.x.

24. Pietrangelo A. Iron in NASH, chronic liver diseases and HCC: how much iron is too much? J Hepatol 2009;50:24951. DOI:10.1016/j.jhep.2008.11.011.

25. Davis RJ, Corvera S, Czech MP. Insulin stimulates cellular iron uptake and causes the redistribution of intracellular transferrin receptors to the plasma membrane. J Biol Chem 1986;261:8708-11.

26. Fabbrini E, deHaseth D, Deivanayagam S, Mohammed BS, VitolaBE, Klein S. Alterations in fatty acid kinetics in obese adolescents with increased intrahepatic triglyceride content. Obesity (Silver Spring) 2009;17:25-9. DOI:10.1038/oby.2008.494.

27. Kirsch R, Sijtsema HP, Tlali M, Marais AD, Hall Pde L. Effects of iron overload in a ratnutritional model of nonalcoholic fatty liver disease. Liver Int 2006;26:1258-67. DOI:10.1111/j.1478-3231.2006.01329.x.

28. McCarty MF. Hyperinsulinemia may boost both hematocrit and iron absorption by up-regulating activity of hypoxiainducible factor-1alpha. Med Hypotheses 2003;61:567-73. DOI:http://dx.doi.org/10.1016/S0306-9877(03)00231-7.

29. Silva M, Silva ME, de Paula H, Carneiro CM, Pedrosa ML. Iron overload alters glucose homeostasis, causes liver steatosis, and increases serum triacylglycerols in rats. Nutr Res 2008;28:391-8. DOI:http://dx.doi.org/10.1016/j.nutres.2008.02.009.

30. Manco M, Bedogni G, Marcellini M, Devito R, Ciampalini P, Sartorelli MR, et al. Waist circumference correlates with liver fibrosis in children with non-alcoholic steatohepatitis. Gut 2008;57:1283-7. DOI:10.1136/gut.2007.142919.

31. Lipinski B, Pretorius E, Oberholzer HM, van der Spuy WJ. Interaction of fibrin with red blood cells: the role of iron. Ultrastruct Pathol 2012;36:79-84. DOI:10.3109/0191312 3.2011.627491.

32. Ferrannini E. Insulin resistance, iron, and the liver. Lancet 2000;355:2181-2. DOI:http://dx.doi.org/10.1016/S01406736(00)02397-7.

33. Matthews DR, Hosker JP, Rudenski AS, Naylor BA, Treacher DF, Turner RC. Homeostasis model assessment: insulin resistance and beta-cell function from fasting plas- ma glucose and insulin concentrations in man. Diabetologia 1985;28:412-9. DOI:10.1007/BF00280883.

34. Choi KM, Lee KW, Kim HY, Seo JA, Kim SG, Kim NH, et al. Association among serum ferritin, alanine aminotransferase levels, and metabolic syndrome in Korean postmenopausal women. Metabolism 2005;54:1510-4. DOI:http://dx.doi.org/10.1016/j.metabol.2005.05.018.

35. Tsimihodimos V, Gazi I, Kalaitzidis R, Elisaf M, Siamopoulos KC. Increased serum ferritin concentrations and liver enzyme activities in patients with metabolic syndrome. Metab Syndr Relat Disord 2006;4:196-203. DOI:10.1089 met.2006.4.196.

36. Sun L, Franco OH, Hu FB, Cai L, Yu Z, Li H, et al. Ferritin concentrations, metabolic syndrome, and type 2 diabetes in middle-aged and elderly chinese. J Clin Endocrinol Metab 2008;93:4690-6. DOI:http://dx.doi.org/10.1210/jc.2008-1159.

37. Gonzalez AS, Guerrero DB, Soto MB, Diaz SP, Martinez-Olmos M, Vidal O. Metabolic syndrome, insulin resistance and the inflammation markers C-reactive protein and ferritin. Eur J Clin Nutr 2006;60:802-9. DOI:10.1038/ sj.ejcn. 1602384 .

38. Jehn M, Clark JM, Guallar E. Serum ferritin and risk of the metabolic syndrome in U.S. adults. Diabetes Care 2004;27:2422-8. DOI:10.2337/diacare.27.10.2422.

39. Freixenet N, Vilardell C, Llaurado G, Gimenez-Palop O, Berlanga E, Gutierrez C, et al. Men with hyperferritinemia and diabetes in the Mediterranean area do not have a higher iron overload than those without diabetes. Diabetes Res Clin Pract 2011;91:e33-6. DOI:http://dx.doi.org/10.1016/j. diabres.2010.10.003. 